\title{
Probing the substrate specificity of the bacterial Pnkp/Hen1 RNA repair system using synthetic RNAs
}

\author{
CAN ZHANG, ${ }^{1}$ CHIO MUI CHAN, ${ }^{1,2}$ PEI WANG, and RAVEN H. HUANG ${ }^{3}$ \\ Department of Biochemistry, University of Illinois at Urbana-Champaign, Urbana, Illinois 61801, USA
}

\begin{abstract}
Ribotoxins cleave essential RNAs involved in protein synthesis as a strategy for cell killing. RNA repair systems exist in nature to counteract the lethal actions of ribotoxins, as first demonstrated by the RNA repair system from bacteriophage T4 25 yr ago. Recently, we found that two bacterial proteins, named Pnkp and Hen1, form a stable complex and are able to repair ribotoxincleaved tRNAs in vitro. However, unlike the well-studied T4 RNA repair system, the natural RNA substrates of the bacterial Pnkp/Hen1 RNA repair system are unknown. Here we present comprehensive RNA repair assays with the recombinant Pnkp/ Hen1 proteins from Anabaena variabilis using a total of 33 different RNAs as substrates that might mimic various damaged forms of RNAs present in living cells. We found that unlike the RNA repair system from bacteriophage T4, the bacterial Pnkp/Hen1 RNA repair system exhibits broad substrate specificity. Based on the experimental data presented here, a model of preferred RNA substrates of the Pnkp/Hen1 repair system is proposed.
\end{abstract}

Keywords: RNA repair; Pnkp/Hen1; substrate specificity; RNA damage; ribotoxin

\section{INTRODUCTION}

RNA in living cells is highly susceptible to damage, primarily due to breakage of the phosphodiester backbone. Phosphodiester bond cleavages are usually triggered by ribonucleases, assisted by the adjacent $2^{\prime} \mathrm{OH}$ group in RNA at the site of breakage, generating $2^{\prime}, 3^{\prime}$-cyclic phosphate at the $3^{\prime}$ end and $5^{\prime} \mathrm{OH}$ at the $5^{\prime}$ end in most cases. Most RNA breakdown is part of normal metabolism, carried out by endogenous ribonucleases. However, cleavage of essential RNAs is exploited by some organisms as a strategy for cell killing. The agents responsible for the RNA cleavage are proteins called ribotoxins, which are usually site-specific ribonucleases. The ribotoxins identified to date kill cells by targeting rRNAs and tRNAs involved in protein translation. Known ribotoxins targeting rRNAs are sarcin (Endo and Wool 1982), restrictocin (Fando et al. 1985), colicin E3 (Bowman et al. 1971), and Biota orientalis ribonuclease (Xu et al. 2004). Several ribotoxins such as colicin E5 (Ogawa

\footnotetext{
${ }^{1}$ These authors contributed equally to this work.

${ }^{2}$ Present address: Division of Environmental and Biomolecular Systems, Oregon Health and Science University, Beaverton, OR 97006, USA.

${ }^{3}$ Corresponding author.

E-mail huang@illinois.edu.

Article published online ahead of print. Article and publication date are at http://www.rnajournal.org/cgi/doi/10.1261/rna.030502.111.
}

et al. 1999), colicin D (Tomita et al. 2000), and Kluyveromyces lactis $\gamma$-toxin ( $\mathrm{Lu}$ et al. 2005) are tRNA-specific ribotoxins, which make a single cut in the anticodon loop of a specific tRNA. In addition to the external ribotoxins that invade cells and damage RNA, a cell sometimes produces its own site-specific ribonucleases to regulate gene expression and cell fate. For example, when infected by bacteriophage T4, some strains of Escherichia coli activate ribonuclease PrrC, which cleaves tRNA ${ }^{\text {Lys }}$ at the wobble position in the anticodon loop (Amitsur et al. 1987). RelE is activated to cleave mRNAs at the ribosome A site to restrict global protein production when a cell is starved (Pedersen et al. 2003; Neubauer et al. 2009). More recently, VapC was shown to have the same effect as RelE but by cleaving the initiator $\mathrm{tRNA}^{\mathrm{fMet}}$ in the anticodon loop (Winther and Gerdes 2011). In eukaryotic organisms, stress causes significant RNA damage, which may involve ribonucleases (Lee and Collins 2005; Thompson et al. 2008; Yamasaki et al. 2009).

Two proteins from bacteriophage T4, Pnkp (polynucleotide kinase-phosphatase) and Rnl1 (RNA ligase 1), counteract the suicidal action of PrrC in infected E. coli by RNA repair (Amitsur et al. 1987). Specifically, bifunctional T4Pnkp hydrolyzes the $2^{\prime}, 3^{\prime}$-cyclic phosphate at the $3^{\prime}$ end and adds a phosphate group at the $5^{\prime}$ end of the cleaved tRNA $^{\text {Lys }}$. Subsequently, T4Rnl1 ligates the two processed 
ends to restore the tRNA ${ }^{\text {Lys }}$ to its original form. Thus, the RNA repair carried out by bacteriophage $\mathrm{T} 4$ precludes disruption of T4 late translation and thus rescues the infection.

Recently, we found that two bacterial proteins repair ribotoxin-cleaved tRNAs in vitro (Chan et al. 2009b). The two proteins, bacterial Pnkp and Hen1, physically interact with each other, forming a heterotetramer in vitro. The bacterial Pnkp from Clostridium thermocellum was first characterized by Martins and Shuman (2005), who showed $5^{\prime}$ kinase, $2^{\prime}, 3^{\prime}$ phosphatase, and adenylyltransferase activities. Interestingly, Pnkp alone could not repair a cleaved RNA (Keppetipola et al. 2007). We demonstrated that RNA repair requires both Pnkp and Hen1 (Chan et al. 2009b). The Pnkp/Hen1 RNA repair system is distinct from the T4Pnkp/Rnl1 system due to the involvement of a fourth enzymatic activity, 2'-O-methyltransferase, which is located at the $\mathrm{C}$ terminus of bacterial Hen1. During RNA repair, bacterial Hen 1 carries out $2^{\prime}$-O-methylation at the $3^{\prime}$ cleavage end after dephosphorylation of the $2^{\prime}, 3^{\prime}$-cyclic phosphate but before ligation of the two cleavage ends (Chan et al. 2009b). Due to the 2'-O-methylation at the junction of repair, the same RNA damage can no longer occur in the repaired RNA.

In contrast to the extensively characterized $\mathrm{T} 4$ system, we know much less about the more recently discovered bacterial Pnkp/Hen1 RNA repair system. Prior studies from the Shuman laboratory and our laboratory have focused on in vitro characterization, either of the Pnkp/Hen1 complex (Chan et al. 2009b) or of components of the complex (Martins and Shuman 2005; Keppetipola et al. 2007; Chan et al. 2009a; Jain and Shuman 2010, 2011). Perhaps the biggest gap is the lack of knowledge about the in vivo RNA substrate(s) of Pnkp/Hen1 and the biological effect of RNA repair in strains containing the Pnkp/Hen1 RNA repair system. If RNA damage is caused internally in bacterial species possessing the Pnkp/Hen1 RNA repair system, it is possible that an in vivo study on one of these bacterial species would provide insight into RNA damage and repair. On the other hand, it is possible that RNA damage in these bacteria could be caused by external agents such as ribotoxins released from other surrounding organisms. If that were the case, then RNA repair would only occur when these bacteria live under conditions that induce such damage, which might not be readily reproduced in a laboratory setting.

Here we present a comprehensive in vitro RNA repair study using Pnkp/Hen1 from Anabaena variabilis (AvaPnkp/ Hen1) and a variety of RNA substrates, with the aim of providing insight into the likely in vivo RNA substrates of the Pnkp/Hen1 RNA repair system. We found that PnkP/ Hen 1 may exhibit broader substrate specificity than T4PnkP/ Rnl1. Based on the studies presented here, the likely in vivo RNA substrates of Pnkp/Hen1 and the possible biological roles of Pnkp/Henl are discussed.

\section{RESULTS}

\section{AvaPnkp/Hen1 efficiently repairs tRNA deletion mutants}

Prior to finding the RNA repair capabilities of bacterial Pnkp/Hen1, several RNA ligase enzymes, with implications for RNA editing or repair, have been biochemically characterized (Amitsur et al. 1987; Ho and Shuman 2002; Simpson et al. 2003; Martins and Shuman 2004a,b). Among them, the T4Pnkp/Rnl1 is the most extensively characterized as an RNA repair system. The in vivo RNA repair target of the T4 system was well defined: to repair E. coli tRNA $^{\text {Lys }}$ cleaved by the endogenous ribonuclease PrrC. Furthermore, the T4Pnkp/Rnll repair system appears to have evolved to optimize RNA repair of the cleaved tRNA; this has been suggested by respective T4Pnkp/tRNA and T4Rnl1/tRNA docking experiments (Galburt et al. 2002; El Omari et al. 2006), as well as a subsequent study by Shuman and coworkers (Nandakumar et al. 2008). Using both the cleaved full-length and tRNA deletion mutants, Shuman and coworkers were able to demonstrate that the efficiency of repair for the cleaved full-length tRNA is significantly higher than the ones for the cleaved tRNA deletion mutants (Nandakumar et al. 2008).

Considering the possibility that like the T4 RNA repair system, the cleaved tRNAs could also be the natural repair targets of the bacterial Pnkp/Hen1 RNA repair system, we performed similar experiments with the AvaPnkp/Hen 1 proteins. The cleaved tRNA Asp and its deletion derivatives were our first choice for the experiments because, like the E. coli $\mathrm{tRNA}^{\text {Lys }}$ cleaved by PrrC, these tRNAs are cleaved by colicin E5 at a position near the wobble nucleotide (Fig. 1). The cleaved full-length RNA $^{\text {Asp }}$ was efficiently repaired by AvaPnkp/Hen1, with a repair yield as high as $65 \%$ after 60 min of repair reaction (Fig. 2A,C, labeled with $\mathrm{tRNA}^{\mathrm{Asp}}$ ). Deletion of the $\mathrm{D}$ stem-loop in $\mathrm{tRNA}^{\mathrm{Asp}}$ resulted in a significantly worse RNA substrate, with a repair yield of $15 \%$ after 60 min of repair reaction (Fig. 2A,C, labeled with tRNA $\left.^{\text {Asp }}-\Delta \mathrm{D}\right)$. Deletion of the T $\Psi \mathrm{C}$ stem-loop was not as bad, with a final repair yield of $40 \%$ (Fig. 2A,C, labeled with tRNA $\left.^{\text {Asp }}-\Delta \mathrm{T}\right)$. Also, AvaPnkp/Hen1 was still able to repair a tRNA ${ }^{\text {Asp }}$ deletion mutant with both $\mathrm{D}$ and T $\Psi \mathrm{C}$ stemloops deleted, with a repair yield comparable to the deletion of $\mathrm{D}$ stem-loop alone (Fig. 2A,C, labeled with $\left.\mathrm{tRNA}^{\text {Asp }}-\Delta \mathrm{DT}\right)$. In addition to the repaired $\mathrm{tRNA}^{\text {Asp }}$ $\Delta \mathrm{DT}$, some ligation products of higher molecular weight (Fig. 2A, marked with ${ }^{\star}$ ) were also produced with the cleaved $\mathrm{tRNA}^{\text {Asp }}-\Delta \mathrm{DT}$ as the substrate. We noticed that $\mathrm{tRNA}^{\text {Asp }}$ $\Delta$ DT-3' has five continuous Gs (Table 1). Therefore, it is possible that repair of abnormal annealed RNA substrates resulted in production of additional ligation products.

Next, we carried out RNA repair of the cleaved tRNA ${ }^{\text {Arg }}$ and its deletion derivatives (Fig. 2B,D). tRNA $^{\text {Arg }}$ and its deletion derivatives were cleaved by colicin $\mathrm{D}$ at a position 

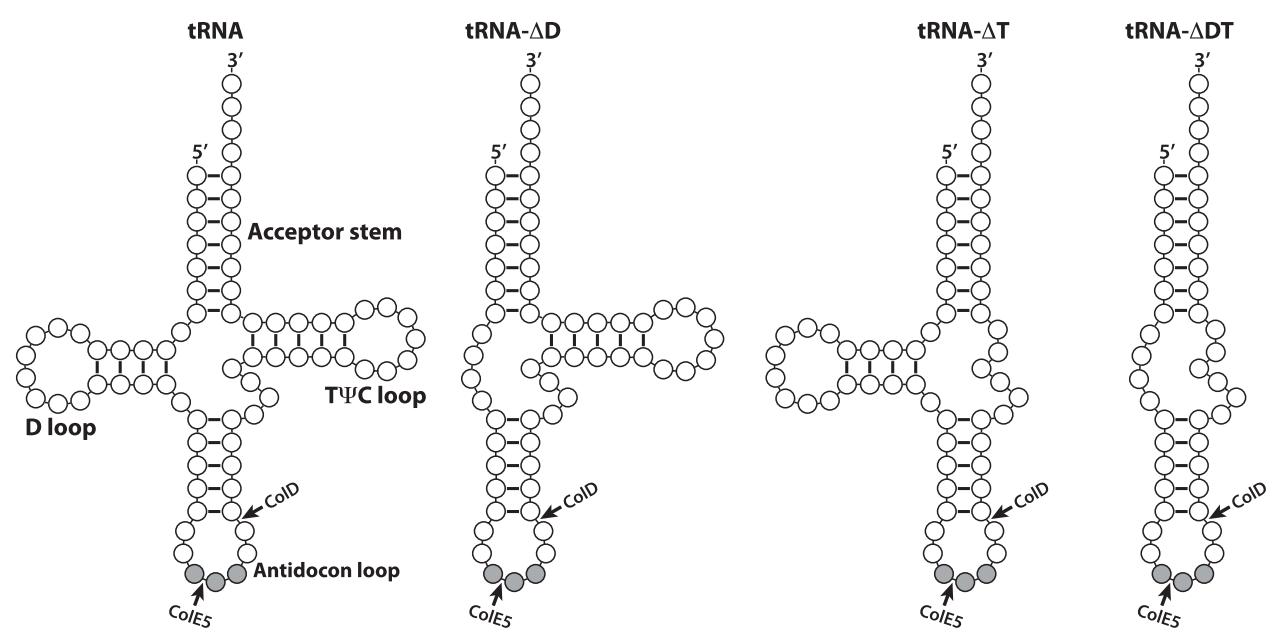

FIGURE 1. Cloverleaf view of full-length and tRNA deletion mutants employed in this study. Nucleotides are depicted as cycles, and they are linked with each other by short thin lines. Base pairs are represented with thick lines. The cleavage sites of tRNA ${ }^{\text {Asp }}$ by colicin E5 (ColE5) and tRNA $^{\text {Arg }}$ by colicin D (ColD) are marked with arrows. Three nucleotides constituting the anticodon of tRNA are highlighted in gray.

that is significantly different from the one by colicin E5 (Fig. 1). Compared with the repair of the cleaved tRNA ${ }^{\text {Asp }}$, cleaved full-length tRNA $^{\text {Arg }}$ was repaired less efficiently, with a repair yield of $30 \%$ after 60 min of repair reaction (Fig. 2B,D, labeled with $\mathrm{tRNA}^{\mathrm{Arg}}$ ). Surprisingly, unlike tRNA $^{\text {Asp }}$ deletion derivatives, deletion of the D stem-loop, $\mathrm{T} \Psi \mathrm{C}$ stem-loop, or both in $\mathrm{tRNA}^{\mathrm{Arg}}$ resulted in RNA substrates that were more efficiently repaired (Fig. 2B,D). The efficiency of repair of these cleaved tRNA ${ }^{\text {Arg }}$ deletion derivatives by $A v a$ Pnkp/Hen 1 is quite remarkable. For example, after only $15 \mathrm{~min}$ of repair reaction, the repair yields of the cleaved tRNA ${ }^{\mathrm{Arg}}-\Delta \mathrm{D}$, $\mathrm{tRNA}^{\mathrm{Arg}}{ }_{-} \Delta \mathrm{T}$, and tRNA ${ }^{\mathrm{Arg}}{ }_{-}$ $\Delta$ DT were $54 \%, 67 \%$, and $53 \%$, respectively (Fig. 2D). At that time point, the repair yields of the cleaved tRNA ${ }^{\text {Asp }}$ and $\mathrm{RRNA}^{\mathrm{Arg}}$ were $22 \%$ and $12 \%$, respectively.

\section{Efficient repair by AvaPnkp/Hen1 requires an RNA substrate of more than a short stem-loop RNA}

Having demonstrated that the drastically deleted tRNAs (e.g., tRNA- $\Delta \mathrm{DT}$ ) could still be effectively repaired by AvaPnkp/Hen1, we next tested if further truncated RNA substrates would be repaired, aiming to define a minimum RNA repair substrate. A total of four additional RNA substrates were prepared for the RNA repair assays. The first two are further deletion of tRNA- $\Delta D T$, with the bulge region between the acceptor stem and anticodon stem deleted (named tRNA- $\Delta \mathrm{DT}-\Delta \mathrm{B}$ ). The deletion of the bulge region produces a stem-loop RNA with a long and continuous stem (Fig. 3A, middle). The other two RNAs correspond to the anticodon stem-loops of tRNA ${ }^{\text {Asp }}$ and tRNA $^{\text {Arg }}$ (named tRNA-AC-SL). To increase the stability of these two RNAs and to make the in vitro repair assay feasible, two additional base pairs were added on the stem (Fig. 3A, left).
Because colicin D did not cleave tRNA ${ }^{\text {Arg }}-\Delta \mathrm{DT}-\Delta \mathrm{B}$ and tRNA $^{\text {Arg }}$-AC-SL, we had to reconstitute these four RNA substrates differently. Specifically, we first prepared the $5^{\prime}$ half and the $3^{\prime}$ half of each RNA substrate individually via chemical synthesis (tRNA-AC-SL) or in vitro transcription (tRNA- $\Delta \mathrm{DT}-\Delta \mathrm{B}$ ), followed by mixing and annealing the two halves. Thus, these RNA substrates do not have the $2^{\prime}, 3^{\prime}$-cyclic phosphate at the $3^{\prime}$ terminus of the $5^{\prime}$ half. To ensure that the missing $2^{\prime}, 3^{\prime}$-cyclic phosphate does not significantly affect the outcome of RNA repair, we used the same approach to prepare tRNA- $\Delta \mathrm{DT}$ substrates as positive controls (Fig. 3A, right).

Each of the six RNA substrates was incubated with either Pnkp-N (containing only the kinase and phosphatase domains of Pnkp) or Pnkp/Hen1 in the presence of ${ }^{33} \mathrm{P}$ $\gamma$-ATP, and the resulting products were analyzed by a denaturing gel (Fig. $3 \mathrm{~B}$ ). The broken tRNA ${ }^{\text {Asp }}-\Delta \mathrm{DT}$ and

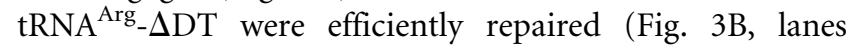
10,12 , cf. with lanes 9,11 , respectively), and their repair yields were comparable to the ones observed previously (Fig. 2), indicating that the lack of the $2^{\prime}, 3^{\prime}$-cyclic phosphate does not significantly affect the outcome of RNA repair. Deleting the bulge in tRNA- $\Delta D T$ has a significant effect on RNA repair. Although a repaired product was produced with the broken tRNA ${ }^{\mathrm{Arg}}{ }_{-} \Delta \mathrm{DT}-\Delta \mathrm{B}$ as the substrate (Fig. 3B, lane 8 marked with an $\left.{ }^{\star}\right)$, its size ( $\sim 29 \mathrm{nt}$ ) did not match the expected size of 35 nt that would have resulted from the ligation of the $5^{\prime}$ half to the $3^{\prime}$ half. Therefore, the identity of the repaired product is unknown. It is possible that, as in the case of tRNA ${ }^{\text {Asp }}-\Delta \mathrm{DT}$ (Fig. 2A), continuous $4 \mathrm{Gs}$ in $\mathrm{tRNA}^{\mathrm{Arg}}-\Delta \mathrm{DT}-\Delta \mathrm{B}-3^{\prime}$ (Table 1 ) could be the culprit of this repaired product. This uncertainty, however, does not affect our general conclusion that the deletion of the bulge region results in an RNA substrate with significantly reduced repair efficiency by $A v a$ Pnkp/Henl (Fig. 3B, cf. lanes 8 

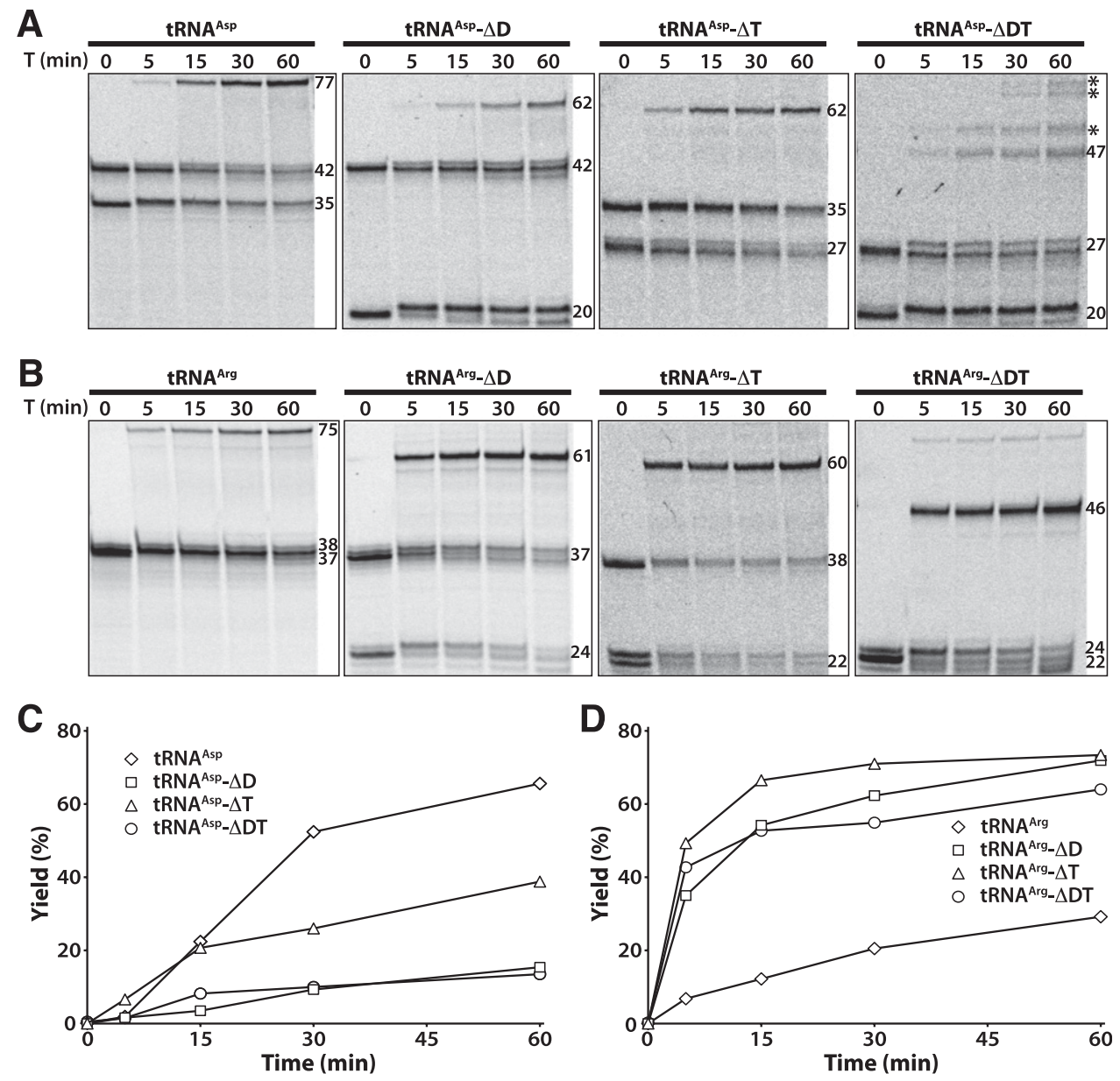

FIGURE 2. Repair of cleaved full-length and tRNA deletion mutants depicted in Figure 1 by AvaPnkp/Hen1. $(A, C)$ Time course of RNA repair of tRNA $^{\text {Asp }}$ and its deletion derivatives. $(B, D)$ Time course of RNA repair of tRNA ${ }^{\text {Arg }}$ and its deletion derivatives. The positions of RNA substrates and repair products are marked with numbers representing their sizes in nucleotides. $\mathrm{T}$ indicates time in minutes.

and 12). Furthermore, deletion of the bulge region in tRNA $^{\text {Asp }}-\Delta$ DT resulted in an RNA substrate that was not repaired at all by $A v a$ Pnkp/Hen1 (Fig. 3B, lane 6). Further shortening the stem of $\mathrm{tRNA}^{\mathrm{Arg}}-\Delta \mathrm{DT}-\Delta \mathrm{B}$ produced an unrepaired RNA substrate (Fig. 3B, lane 4), and like tRNA $^{\text {Asp }}-\Delta D T-\Delta B$, tRNA ${ }^{\text {Asp }}-$ AC-SL was not a substrate of AvaPnkp/Hen1 (Fig. 3B, lane 2).

\section{AvaPnkp/Hen1 does not efficiently repair nicked and bulged double-stranded RNAs}

The single-stranded regions of RNAs are the most susceptible to RNA damage in vivo. Indeed, all ribotoxins discovered to date target single-stranded RNAs (ssRNAs) for cleavage. In addition to pure ssRNAs and loops of the stem-loop RNAs, bulges that interrupt continuation of double-stranded RNAs are also frequently present as ssRNAs in living cells. Therefore, we decided to carry out an investigation to see if $A v a$ Pnkp/Hen1 was able to repair RNAs damaged in the bulge region. Furthermore, we were also interested in knowing if $A v a P n k p / H e n 1$ could repair a nicked double-stranded RNA.

Our strategy for substrate preparation of the study described in this section was to utilize two ssRNAs available in our laboratory (named Lead and Tail RNAs, respectively) as the targets of repair. These two ssRNAs were brought together by base-pairing with a complementary strand (named Bridge). By varying the nucleotide sequence of Bridge RNA, four RNA repair substrates differing in their secondary structures were produced (Fig. 4A-D). To simplify interpretation of repair results, only Tail RNA was radiolabeled (Fig. 4, marked with a $\left.{ }^{\star}\right)$. These four RNA substrates, together with other various combinations of Tail, Lead, and Bridge RNAs (used as negative controls), were incubated with AvaPnkp/ Henl for RNA repair and the results were surprising (Fig. 4E).

First, only a small amount of the repaired products was produced with the nicked and bulged RNA substrates (Fig. 4E, lanes 8-11), indicating that, unlike the cleaved stemloop RNAs, RNAs damaged within the double-stranded region (nicked RNA) (Fig. 4A) or in the bulge region of the 
TABLE 1. RNAs used in this study

\begin{tabular}{|c|c|c|}
\hline RNA & Sequence $\left(5^{\prime}-3^{\prime}\right)$ & Size (nt) \\
\hline tRNA ${ }^{\text {Asp }}$ & $\begin{array}{l}\text { GGA GCG GUA GUU CAG UCG GUU AGA AUA CCU GCC UGU CAC GCA GGG GGU CGC } \\
\text { GGG UUC GAG UCC CGU CCG UUC CGC CA }\end{array}$ & 77 \\
\hline tRNA ${ }^{\text {Asp }}-\Delta \mathrm{D}$ & $\begin{array}{l}\text { GGA GCG GUA GUA CCU GCC UGU CAC GCA GGG GGU CGC GGG UUC GAG UCC CGU } \\
\text { CCG UUC CGC CA }\end{array}$ & 62 \\
\hline tRNA ${ }^{\text {Asp }}-\Delta T$ & $\begin{array}{l}\text { GGA GCG GUA GUU CAG UCG GUU AGA AUA CCU GCC UGU CAC GCA GGG GGU CGU } \\
\text { CCG UUC CGC CA }\end{array}$ & 62 \\
\hline tRNA ${ }^{\text {Asp }}-\Delta \mathrm{DT}$ & GGA GCG GUA GUA CCU GCC UGU CAC GCA GGG GGU CGU CCG UUC CGC CA & 47 \\
\hline tRNA $^{\text {Arg }}$ & $\begin{array}{l}\text { GUC CUC UUA GUU AAA UGG AUA UAA CGA GCC CCU CCU AAG } \\
\text { GGC UAA UUG CAG GUU CGA UUC CUG CAG GGG ACA CCA }\end{array}$ & 75 \\
\hline tRNA ${ }^{A r g}-\Delta \mathrm{D}$ & $\begin{array}{l}\text { GUC CUC UUA GCG AGC CCC UCC UAA GGG CUA AUU GCA GGU UCG AUU CCU GCA } \\
\text { GGG GAC ACC A }\end{array}$ & 61 \\
\hline $\operatorname{tRNA}{ }^{\mathrm{Arg}}-\Delta \mathrm{T}$ & $\begin{array}{l}\text { GUC CUC UUA GUU AAA UGG AUA UAA CGA GCC CCU CCU } \\
\text { AAG GGC UAA UUG CAG GGG ACA CCA }\end{array}$ & 60 \\
\hline tRNA ${ }^{\text {Arg }}-\Delta \mathrm{DT}$ & GUC CUC UUA GCG AGC CCC UCC UAA GGG CUA AUU GCA GGG GAC ACC A & 46 \\
\hline tRNA ${ }^{\text {Asp }}-\Delta D^{\prime} T^{\prime}$ & GGA GCG GUA GUA CCU GCC UG & 20 \\
\hline tRNA ${ }^{\text {Asp }}-\Delta \mathrm{DT}-3^{\prime}$ & UCA CGC AGG GGG UCG UCC GUU CCG CCA & 27 \\
\hline tRNA ${ }^{\text {Arg }}-\Delta \mathrm{DT}-5^{\prime}$ & GUC CUC UUA GCG AGC CCC UCC UAA & 24 \\
\hline tRNA ${ }^{\text {Arg }}-\Delta \mathrm{DT}-3^{\prime}$ & GGG CUA AUU GCA GGG GAC ACC A & 22 \\
\hline tRNA ${ }^{A s p}-\Delta D T-\Delta B-5^{\prime}$ & GGA GCG GCC UGC CUG & 15 \\
\hline tRNA ${ }^{\text {Asp }}-\Delta \mathrm{DT}-\Delta \mathrm{B}-3^{\prime}$ & UCA CGC AGG CCG UUC CGC CA & 20 \\
\hline tRNA ${ }^{\text {Arg }}-\Delta$ DT- $\Delta \mathrm{B}-5^{\prime}$ & GUC CUC UAG CCC CUC CUA A & 19 \\
\hline tRNA ${ }^{\text {Arg }}-\Delta$ DT- $\Delta B-3^{\prime}$ & GGG CUA GGG GAC ACC A & 16 \\
\hline tRNA ${ }^{\text {Asp }}-$ AC-SL-5' & CGC CUG CCU G & 10 \\
\hline tRNA ${ }^{\text {Asp }}-$ AC-SL-3' & UCA CGC AGG CG & 11 \\
\hline tRNA ${ }^{\text {Arg }}-\mathrm{AC}-\mathrm{SL}-5^{\prime}$ & GCA GCC CCU CCU AA & 14 \\
\hline tRNA ${ }^{\text {Arg }}-A C-S L-3^{\prime}$ & GGG CUG C & 7 \\
\hline Lead & UAU GCG AUG CUU GCU GAC GGA CUA ACU & 27 \\
\hline Tail & GGC CCG UUA GUC AAG GAG UUA AGA CAC CAC & 30 \\
\hline Bridge1 & $\begin{array}{l}\text { GUG GUG UCU UAA CUG CUU GAC UAA CGG GCC AGU UAG UCC GUC AGC AAG } \\
\text { CAU CGC AUA }\end{array}$ & 57 \\
\hline Bridge2 & GUG GUG UCU UAA CUG CUU GAC UAA CGA GUC CGU CAG CAA GCA UCG CAU A & 49 \\
\hline Bridge3 & GUG GUG UCU UAA CUG CUU GAC UAA CGA AAA AGU CCG UCA GCA AGC AUC GCA UA & 53 \\
\hline Bridge4 & $\begin{array}{l}\text { GUG GUG UCU UAA CUG CUU GAC UAA CGA AAA UAC CAG UCC GUC AGC AAG } \\
\text { CAU CGC AUA }\end{array}$ & 57 \\
\hline S1 & UGC GAU GCU UGC UGA CGG ACU & 21 \\
\hline S2 & UGC GAU GCU UGC UGA CGG ACU AAU & 24 \\
\hline S3 & UAG UCC GUC AGC AAG CAU CGC AUA & 24 \\
\hline S4 & GGC ACG GCU GUA AAC CGU GC & 20 \\
\hline S5 & GCC GGC UGU AAA CCG GC & 17 \\
\hline S6 & GGG UGC UCA GUA CGA GAG GAA CCG CAC CC & 29 \\
\hline S7 & GGA GCG GUA GUU CAG UCG GUU AGA AUA CCU GCC UG & 35 \\
\hline
\end{tabular}

double-stranded RNA cannot be effectively repaired by AvaPnkp/Hen1.

Efficient RNA repair was observed with some RNA substrates originally designed as negative controls. Specifically, in the absence of Lead RNA, Tail RNA was efficiently ligated to Bridge3 and Bridge4 RNAs (Fig. 4E, lanes 6,7), but not Bridge1 and Bridge2 RNAs (Fig. 4E, lanes 4,5). Without Lead RNA, Tail RNA and Bridge3 or Bridge4 RNAs form a double-stranded RNA with a 4-nt single-stranded 5' overhang of Tail RNA, and a 27-nt or 31-nt single-stranded $3^{\prime}$ overhang of Bridge3 or Bridge4 (Supplemental Fig. S1C,D). Efficient RNA repair of Tail/Bridge3 and Tail/ Bridge 4 combinations indicates ligation of the 5 ' overhang of Tail RNA to the $3^{\prime}$ overhang of the Bridge 3 or Bridge 4 RNAs to form a 31-nt or 35-nt size loop. The inability of
Tail RNA to be ligated to Bridge1 (Fig. 4E, lane 4) can be explained by the lack of the flexible single-stranded 5' overhang of Tail RNA in the Tail/Bridgel combination (Supplemental Fig. S1A). On the other hand, the negative result of RNA repair in the Tail/Bridge 2 combination was initially puzzling, as it should have produced a result similar to the Tail/Bridge3 combination because they appeared to have similar single-stranded $5^{\prime}$ and $3^{\prime}$ overhangs (Fig. 4B,C). Further inspection of the nucleotide sequences of these two Bridge RNAs explains why. In the absence of Lead RNA, three of the four nucleotides in Bridge 2 originally designed to base-pair with the $3^{\prime}$ end of Lead RNA are now able to base-pair with the single-stranded $5^{\prime}$ overhang of Tail RNA (Supplemental Fig. S1B). In essence, the Tail/Bridge2 combination has a secondary structure similar to the one of the 
A
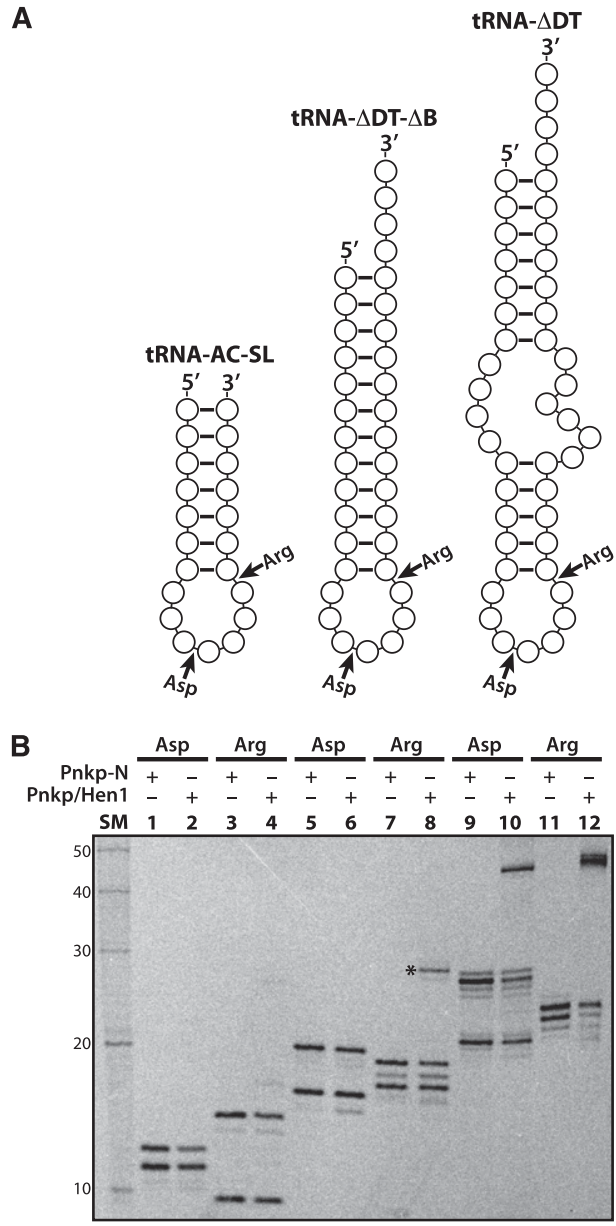

FIGURE 3. Repair of broken stem-loop RNAs by AvaPnkp/Hen 1 . (A) Schematic view of three sets of broken stem-loop RNAs used for the assay. Since each substrate is reconstituted by mixing and annealing separately prepared $5^{\prime}$-half and $3^{\prime}$-half RNAs, its breaking point is marked with an arrow and labeled as Asp (for nucleotide sequence matching tRNA ${ }^{\text {Asp }}$ ) or Arg (for nucleotide sequence matching tRNA ${ }^{\mathrm{Arg}}$ ). (B) Analysis of phosphorylation and repair reactions by DPAGE. Pnkp-N indicates the N-terminal half of Pnkp consisting of the kinase and phosphatase domains. SM indicates RNA size marker.

Tail/Bridge1 combination (Supplemental Fig. S1B, cf. with Supplemental Fig. S1A). It is therefore only logical that RNA repair of these two combinations by AvaPnkp/Hen1 produced similar results (Fig. 4E, lanes 4,5).

Another observation was the efficient ligation of Tail RNA to Lead RNA in the absence of any Bridge RNAs (Fig. $4 \mathrm{E}$, lane 3). Again, inspection of the nucleotide sequences of these two RNAs provides rationale as to why these two RNAs were efficiently ligated by AvaPnkp/Hen1. Within a region of $11 \mathrm{nt}$ near the $3^{\prime}$ end of Lead RNA and the $5^{\prime}$ end of Tail RNA, these two RNAs are able to form a duplex of a total of $10 \mathrm{bp}$, and eight of them are continuous (Supplemental Fig. S2A). Base-pairing between Lead RNA and Tail RNA produces an RNA substrate with a 12-nt single-stranded $5^{\prime}$ overhang of Lead RNA and a 19-nt single-stranded 3' overhang of Tail RNA. Presumably, these two ends were ligated by AvaPnkp/Hen 1 during RNA repair, producing a stem-loop RNA with a 31-nt size loop.

In addition to the efficient RNA repair of the Tail/Lead, Tail/Bridge3, and Tail/Bridg4 combinations, a small amount of self-ligated product of Tail RNA was also observed in the absence of other RNAs (Fig. 4E, lane 2). A possible explanation for this inefficient self-ligation is the potential formation of four GC pairs via $5^{\prime}$ ends of two Tail RNAs.

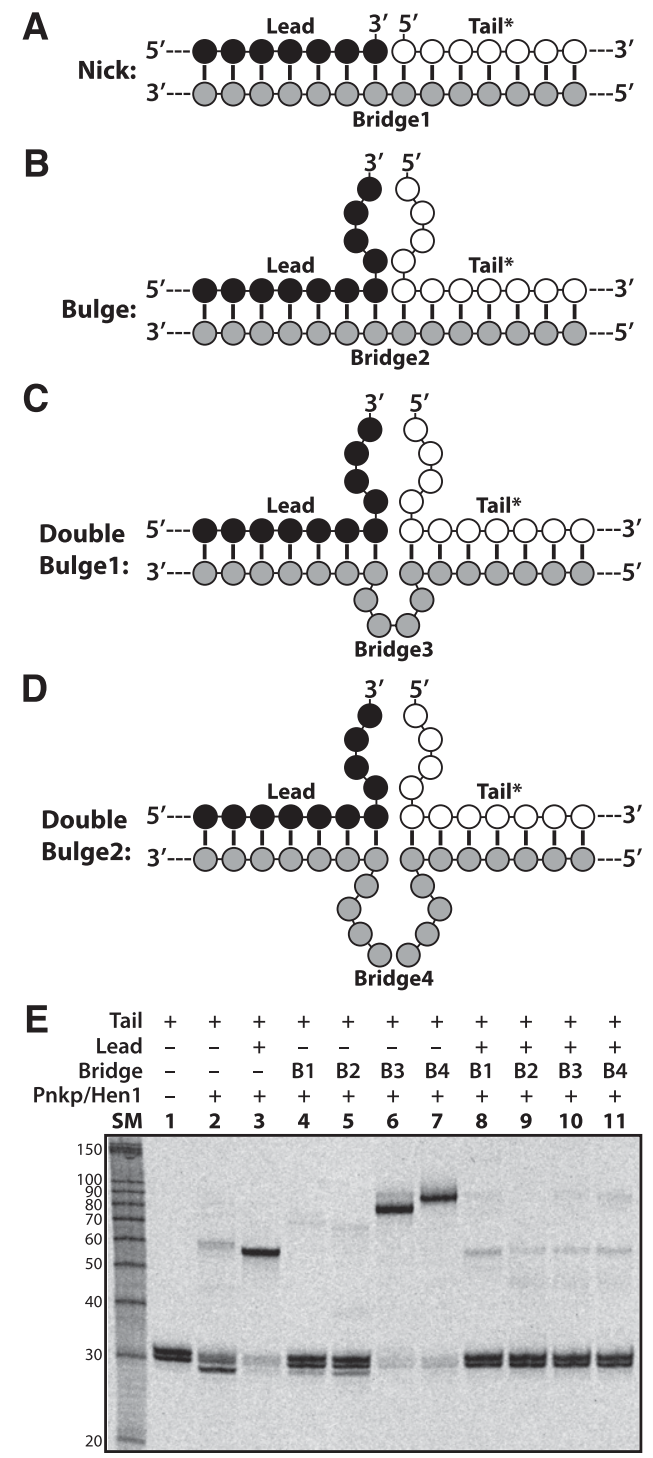

FIGURE 4. Repair of broken double-stranded RNAs by AvaPnkp/ Hen1. $(A-D)$ Schematic depiction of different secondary structures of RNA repair substrates with single RNA break in the regions of doublestranded RNA (Nick) and bulges (Bulge, Double Bulge1, and Double Bulge2). Only Tail RNA, which is depicted with white cycles, is radiolabeled (with ${ }^{\star}$ ). Lead RNA, the potential repair partner of Tail, is colored black, and the complementary strand bridging Tail and Lead RNAs (Bridge1 to Bridge4) is in gray. (E) Analysis of RNA repair products by DPAGE. B1 to B4 indicate Bridgel to Bridge4. 


\section{AvaPnkp/Hen1 does not ligate two unrelated ssRNAs}

Due to the unexpected result of RNA repair of the Tail/ Lead combination (Fig. 4E, lane 3), we decided to further investigate potential RNA ligation of Tail RNA with other ssRNAs available in our laboratory. We have chosen seven ssRNAs (Table 1, S1 to S7) for the study. Based on their nucleotide sequences, these seven ssRNAs can be divided into three groups. S1 and S2 RNAs belong to the first group, both of which are related to Lead RNA due to their high nucleotide sequence identities (Supplemental Fig. S2B). Indeed, like Lead RNA, S1 and S2 RNAs were efficiently ligated to Tail RNA by AvaPnkp/Hen1 (Fig. 5, lanes 4,5, cf. lane 3). The positions of the ligation products in the gel are consistent with the expected ligation sizes of Lead (27 nt), S1 (21 $\mathrm{nt}$ ), and S2 (24 nt) RNAs to Tail RNA (30 nt).

S3 is the only RNA that belongs to the second group. S3 is also related to Lead RNA. But instead of sharing the same nucleotide sequences with Lead RNA as S1 and S2, S3 is complementary to Lead RNA (Supplemental Fig. S2C). RNA ligation of $\mathrm{S} 3$ to Tail RNA was not observed (Fig. 5, lane 6). Therefore, the positive results from $\mathrm{S} 1$ and $\mathrm{S} 2$, and the negative result from $\mathrm{S} 3$, provide further support for the explanation that efficient ligation of Tail RNA to Lead RNA is due to formation of a secondary structure as depicted in Supplemental Figure S2A.

RNAs in the third group are S4, S5, S6, and S7 (ranging in size from 17-35 nt) (Table 1). Based on their nucleotide sequences, these RNAs are not related to Lead RNA or to Tail RNA. None of them were able to ligate to Tail RNA (Fig. 5, lanes 7-10). Therefore, we conclude that if two ssRNAs do not physically interact with each other via basepairing to bring them together, their ligation by AvaPnkp/ Hen1 will not occur.

It is interesting to notice that whereas a small amount of self-ligated product was produced with Tail RNA alone (Fig. 5, lane 2), such a ligation product was not observed with the addition of S3-S7 (Fig. 5, lanes 6-10). Presumably, the addition of excess ssRNAs S3-S7 sequesters a significant

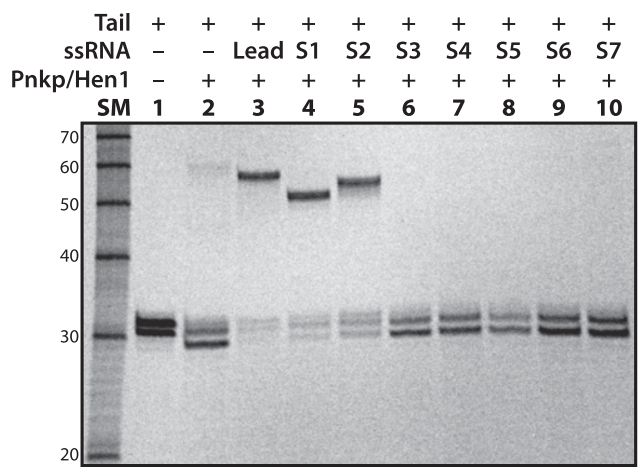

FIGURE 5. Repair of RNA substrates consisting of two singlestranded RNAs (ssRNAs) by AvaPnkp/Hen1. As in the case of Figure 4 , only Tail RNA is radiolabeled. amount of enzyme, making inefficient self-ligation of Tail RNA unlikely to occur.

\section{DISCUSSION}

Based on results of RNA repair assays presented in this study, we summarize an effective RNA substrate for repair by $A v a$ Pnkp/Hen1 as schematically depicted in Figure 6.

To be an effective RNA repair substrate of $A v a P n k p / H e n 1$, two elements are essential. The first element is the two singlestranded ends from the original RNA cleavage, which are the repair targets and are depicted here as $5^{\prime}$ and $3^{\prime}$ overhangs (Fig. 6). The second element is a means to bring these two ends together to facilitate repair by AvaPnkp/ Hen1. Our data indicate that the lengths of both $5^{\prime}$ and $3^{\prime}$ overhangs are quite flexible, and a stable stem is the most effective method to bring these two overhangs together for efficient repair.

For the $5^{\prime}$ overhang, we have observed efficient repair of RNA substrates with its length from 4-12 nt (Fig. 4E, lanes 3,6,7; Supplemental Figs. S1C,D, S2A). It is likely that RNA substrates with the $5^{\prime}$ overhang $>12 \mathrm{nt}$ can also be efficiently repaired by AvaPnkp/Hen1, but it requires future experiments for confirmation. The shortest length of $5^{\prime}$ overhang allowed for effective repair is in fact zero, demonstrated by the effective repair of the cleaved tRNA ${ }^{\mathrm{Arg}}$ and its truncated derivatives (Figs. 1, 2). However, tRNA ${ }^{\mathrm{Arg}}$ and its deletion derivatives are likely to be an exception as similar configurations in the Tail/Bridge1 and Tail/Bridge2 combinations did not work well for repair (Fig. 4E, lanes 4,5; Supplemental Fig. S1A,B). It is possible that, after cleavage by colicin D, the $5^{\prime}$-terminal nucleotide (nucleotide 39) in tRNA ${ }^{\mathrm{Arg}}$ and its deletion derivatives might no longer base-pair with its partner (nucleotide 31 ) due to limited stability of the anticodon stem $\left(5 \mathrm{bp}\right.$ ). This would provide the $5^{\prime}$ end some flexibility for the repair reactions (phosphorylation and ligation) to be carried out. This hypothesis is consistent with the observations that the cleaved RRNA $^{\text {Arg }}$ deletion mutant is repaired more efficiently than the full-length tRNA ${ }^{\mathrm{Arg}}$ (Fig. 2B,D). Deleting the D or/and TYC stem-loop in tRNA ${ }^{\text {Arg }}$ would likely produce less stable RNAs, which might be better substrates of $A v a$ Pnkp/Hen1 due to more potential flexibility of the 5'-terminal nucleotide. Conversely, because both Tail/ Bridgel and Tail/Bridge2 have long stems (Supplemental Fig. S1A,B), the flexibility of the $5^{\prime}$-terminal nucleotide amenable for RNA repair seen in tRNA ${ }^{\mathrm{Arg}}$ and its deletion derivatives might not be present in the Tail/Bridgel and Tail/ Bridge2 combinations, resulting in inefficient repair of Tail/ Bridge1 and Tail/Bridge2 (Fig. 4E, lanes 4,5).

For the $3^{\prime}$ overhang, RNA substrates with its length ranging from $3 \mathrm{nt}$ (tRNA ${ }^{\text {Asp }}$ and its derivative) (Fig. 2A,C) to 31 nt (Tail/Bridge4 combination) (Fig. 4E, lane 7) were seen to be efficiently repaired by AvaPnkp/Hen1. It is possible that the $3^{\prime}$ overhang could even be $<3$ nt, but we have not tested this possibility. 


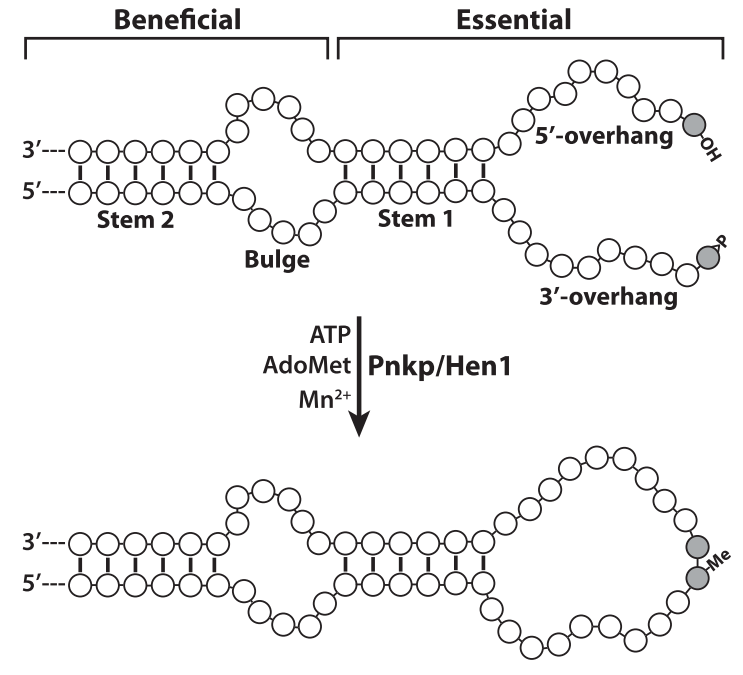

FIGURE 6. Schematic depiction of an effective RNA substrate of AvaPnkp/Hen1 and its repaired product based on experimental data presented in this study. The two terminal nucleotides to be ligated by AvaPnkp/Henl are highlighted in gray, with the depicted 5'-OH and $2^{\prime}, 3^{\prime}$-cyclic phosphate groups that are directly involved in repair chemistry. ATP is required for phosphorylation of the $5^{\prime}-\mathrm{OH}$ group, and AdoMet is the methyl donor for $2^{\prime}$-O-methylation on the $3^{\prime}$ terminal nucleotide during repair, which requires a manganese ion $\left(\mathrm{Mn}^{2+}\right)$ to carry out the reaction. The numbers of nucleotides for the different regions of RNA (5' overhang, 3' overhang, Stem 1, Bulge, and Stem 2) were chosen arbitrarily. Secondary structures of the Bulge, the $5^{\prime}$ overhang, the $3^{\prime}$ overhang, and the resulting repaired loop were also arbitrary depicted.

We propose that a stable stem, as depicted Stem 1 in Figure 6 , is the best means to bring two overhangs together for efficient repair by AvaPnkp/Hen 1 based on the following observations. First, AvaPnkp/Hen 1 is unable to repair an RNA substrate that does not form a secondary structure to bring the two ends together. This is supported by the negative results of ligating Tail RNA to other unrelated ssRNAs (Fig. 5, lanes 6-10). Second, employing two double-stranded RNAs to bring the two ends together for repair did not work well, as AvaPnkp/Hen1 was unable to repair the damaged bulged RNAs (Fig. 4E, lanes 8-11). Third, of the all the RNA substrates we have tested for repair so far, RNAs that were efficiently repaired by AvaPnkp/Hen1 are always having secondary structures of some variations of the cleaved stem-loop RNAs (tRNA ${ }^{\text {Asp }}$ and its deletion derivatives, tRNA $^{\text {Arg }}$ and its deletion derivatives, Tail/Lead combination, Tail/Bridge3 combination, and Tail/Bridge4 combination). The discrimination of the cleaved stem-loop RNAs vs. the cleaved bulged RNAs for repair by AvaPnkp/ Hen 1 might reflect the structure of RNA-binding groove/ pocket in the Pnkp/Hen1 complex. It is possible that the RNA binding groove/pocket of Pnkp/Hen1 is not suitable for binding of a damaged bulged RNA, which requires accommodation of two double-stranded RNAs on both sides of the cleaved bulge RNA. A crystal structure of the Pnkp/Hen1 complex should confirm or refute this possibility.

In addition to the two essential elements described above, other RNA elements beyond the required stem might be beneficial, and in some cases are essential, for efficient RNA repair (Fig. 6). While tRNA- $\Delta$ DT was efficiently repaired, deletion of the bulge region in tRNA- $\Delta$ DT produced RNAs that were not good substrates of $A v a$ Pnkp/Hen1 (Fig. 3). We postulate that some single-stranded elements in RNA substrates are needed to provide sufficient interaction with Pnkp/Hen 1 for efficient repair. Cleaved tRNA- $\Delta$ DT has the bulge region to provide such elements. Although Tail/ Bridge3, Tail/Bridge4, and Tail/Lead combinations do not have bulges (Supplemental Figs. S1C,D, S2A), they have long single-stranded overhangs as the single-stranded elements. On the other hand, cleaved tRNA- $\Delta \mathrm{DT}-\Delta \mathrm{B}$ and tRNA-AC-SL do not have bulges, and their $5^{\prime}$ and $3^{\prime}$ overhangs are too short to be the providers of the single-stranded elements. Again, a crystal structure of Pnkp/Hen1, which we are actively working on, should provide insight into whether interaction with the single-stranded region of an RNA substrate by Pnkp/Hen1 is required for efficient RNA repair.

As we have discussed previously, the biological roles of Pnkp/Hen1 in vivo are unknown. Nevertheless, we could speculate about the possible situations within living cells where RNA repair is required. If RNA damage in bacteria carrying Pnkp/Hen1 is induced by exogenous agents, we could then imagine two possible scenarios in which a bacterium requires RNA repair. The first is in an emergency, such as when a bacterium is invaded by a ribotoxin. A ribotoxin could cleave an essential RNA so rapidly that new rounds of transcription might not be fast enough to save the cell. This scenario requires only a short-term solution, and bacterial Pnkp/Hen1 could play such a role. Evidence supporting such a possibility is shown by more efficient repair of the cleaved full-length tRNA ${ }^{\text {Asp }}$ compared with the cleaved tRNA ${ }^{\text {Asp }}$ deletion derivatives (Fig. $2 \mathrm{~A}, \mathrm{C})$. However, the difference in efficiency of repairing the cleaved full-length tRNA ${ }^{\text {Asp }}$ vs. the deletion derivative appears to be much smaller in the bacterial Pnkp/Hen1 system than the T4Pnkp/Rnll system (Nandakumar et al. 2008). This observation, together with the more efficient RNA repair of the tRNA ${ }^{\text {Arg }}$ deletion mutant vs. the fulllength one, is consistent with the Pnkp/Henl employed for RNA repair in a second scenario described below.

In a second scenario, repair of damaged RNAs might be employed as a long-term strategy to "improve the quality of life" of bacteria that suffer constant RNA damage. In such a case, the damaged RNAs need to be repaired in such a way that the RNAs after repair could no longer be cleaved again. Otherwise, the cycle of RNA damage followed by RNA repair would keep repeating. We have previously demonstrated that, because of the $2^{\prime}-\mathrm{O}$ methylation at the junction of RNA repair, repaired RNA can no longer be cleaved again at the site of repair (Chan et al. 2009b). The data presented in this 
study indicate that Pnkp/Hen 1 is able to repair a variety of damaged RNAs with high efficiency. Therefore, the mechanism of RNA repair carried out by Pnkp/Hen1 and the broad RNA substrate specificity of AvaPnkp/Hen1 described in this study are consistent with the second scenario. From a logical point of view, how an RNA is damaged is not the concern of the Pnkp/Hen1 RNA repair system. The presence of a significant amount of a particular RNA cleaved at a specific site is sufficient to indicate the vulnerability of the site. One round of repair of the cleaved RNA by Pnkp/Hen1 with the $2^{\prime}$-O-methylation at the repair junction helps the cell solve the RNA damage problem at that particular site.

We believe that the general conclusions based on our in vitro RNA repair study of Pnkp/Hen1 from A. variabilis are applicable for Pnkp/Hen1 from other bacterial species. However, we cannot rule out the possibility that, during the long history of bacterial evolution, Pnkp/Henl in different bacterial species have maintained the same chemistry for RNA repair (5'-phosphorylation, $3^{\prime}$-dephosphorylation, 5',3'-ligation, and $2^{\prime}$-O-methylation) but have evolved different RNA substrate specificity due to the requirement of repairing specific RNA damages caused by RNA-damaging agents present in their local living environments. Future studies similar to the one presented here, but using Pnkp/Hen1 from other organisms, should address this issue. In addition, we have recently found a second subfamily of the bacterial Pnkp/Hen1 RNA repair system that is significantly different from the first subfamily in the amino acid sequences of both Pnkp and Hen1 (data not shown). Therefore, it is possible that Pnkp/Hen 1 from the second subfamily might have a different RNA substrate specificity from those in the first subfamily, of which AvaPnkp/Hen1 is a member.

\section{MATERIALS AND METHODS}

\section{Recombinant proteins}

AvaPnkp/Hen 1 and AvaPnkp-N were overexpressed in E. coli BL21(DE3) and purified from soluble bacterial lysates as described previously (Chan et al. 2009b).

\section{Preparation of full-length and cleaved RNAs}

All the RNAs listed in Table 1 were in vitro transcribed using T7 polymerase with the exception of tRNA ${ }^{\text {Asp }}-\mathrm{AC}_{-} \mathrm{SL}-5^{\prime}$, $\mathrm{tRNA}^{\mathrm{Asp}}$ AC-SL-3', tRNA ${ }^{\text {Arg }}$-AC-SL-5', tRNA ${ }^{\text {Arg }}$-AC-SL-3', Lead, S1, S2, S3, S4, and S5 RNAs, which were purchased from IDT. Both in vitro transcribed and purchased RNAs were purified by DPAGE. In vitro transcription and cleavage of tRNAs by colicin E5 and colicin D were carried out as described previously (Chan et al. 2009b).

\section{RNA repair assays with $t R N A s$ and its deletion derivatives RNAs}

The RNA repair reaction was carried out in a reaction mixture containing $25 \mathrm{mM}$ Tris- $\mathrm{HCl}(\mathrm{pH} 8.0), 50 \mathrm{mM} \mathrm{KCl}, 2.5 \mathrm{mM}$
$\mathrm{MgCl}_{2}, 0.05 \mathrm{mM}$ EDTA, 2.5\% glycerol, $5 \mathrm{mM}$ DTT, $0.2 \mathrm{mM}$ ATP, $0.5 \mathrm{mM} \mathrm{MnCl}, 0.05 \mathrm{mM}$ S-AdoMet, $1.0 \mu \mathrm{M}$ internally ${ }^{33} \mathrm{P}-$ radiolabeled cleaved tRNA or its deletion derivatives, and $0.5 \mu \mathrm{M}$ of $A v a \mathrm{Pnkp} / \mathrm{Hen} 1$ heterotetramer. The reaction mixture was incubated for $0,5,15,30$, and $60 \mathrm{~min}$ at $37^{\circ} \mathrm{C}$. The reactions were quenched by adding DPAGE loading buffer. The samples were heated for $3 \mathrm{~min}$ at $95^{\circ} \mathrm{C}$ and analyzed by $15 \%$ DPAGE. The radioactivity of the repaired and unrepaired tRNAs was quantified using a PhosphorImager system (Molecular Dynamics).

\section{RNA repair assays with stem-loop RNAs}

The RNA repair reaction was carried out in a reaction mixture containing $25 \mathrm{mM}$ Tris- $\mathrm{HCl}(\mathrm{pH} 8.0), 50 \mathrm{mM} \mathrm{KCl}, 2.5 \mathrm{mM}$ $\mathrm{MgCl}_{2}, 0.05 \mathrm{mM}$ EDTA, $2.5 \%$ glycerol, $5 \mathrm{mM}$ DTT, $0.5 \mathrm{mM}$ $\mathrm{MnCl}_{2}, 0.05 \mathrm{mM}$ S-AdoMet, $1 \mu \mathrm{M}$ of an RNA substrate (tRNAAC-SL, tRNA- $\Delta$ DT- $\Delta$ B, or tRNA- $\Delta \mathrm{DT}$ ), and $0.5 \mu \mathrm{M}$ of $A v a$ Pnkp/ Hen 1 or AvaPnkp-N in the presence of $0.2 \mathrm{mM} \mathrm{ATP} /{ }^{33} \mathrm{P}-\gamma$-ATP mixture. The reaction mixture was incubated for $40 \mathrm{~min}$ at $37^{\circ} \mathrm{C}$. The samples were heated for $3 \mathrm{~min}$ at $95^{\circ} \mathrm{C}$ and analyzed by $20 \%$ DPAGE. The radioactivity of the repaired and unrepaired tRNAs was quantified using a PhosphorImager system (Molecular Dynamics).

\section{RNA repair assays with the nicked and broken bulged double-stranded RNAs and with ssRNAs}

The RNA repair reaction was carried out in a reaction mixture containing $25 \mathrm{mM}$ Tris- $\mathrm{HCl}(\mathrm{pH} 8.0), 50 \mathrm{mM} \mathrm{KCl}, 2.5 \mathrm{mM}$ $\mathrm{MgCl}_{2}, 0.05 \mathrm{mM}$ EDTA, 2.5\% glycerol, $5 \mathrm{mM}$ DTT, $0.2 \mathrm{mM}$ ATP, $0.5 \mathrm{mM} \mathrm{MnCl}_{2}, 0.05 \mathrm{mM}$ S-AdoMet, $1 \mu \mathrm{M}$ of an RNA substrate with only Tail RNA internally radiolabeled with ${ }^{33} \mathrm{P}-\alpha$-ATP, and $0.5 \mu \mathrm{M}$ of $A v a$ Pnkp/Hen 1 heterotetramer. The reaction mixture was incubated for $40 \mathrm{~min}$ at $37^{\circ} \mathrm{C}$. The reactions were quenched by adding DPAGE loading buffer. The samples were heated for 3 min at $95^{\circ} \mathrm{C}$ and analyzed by $15 \%$ DPAGE. The radioactivity of the repaired and unrepaired tRNAs was quantified using a PhosphorImager system (Molecular Dynamics).

\section{SUPPLEMENTAL MATERIAL}

Supplemental material is available for this article.

\section{ACKNOWLEDGMENTS}

We thank K. Selvadurai for critical reading of the manuscript. This research was supported by NSF grant MCB-0920966.

Received September 23, 2011; accepted November 9, 2011.

\section{REFERENCES}

Amitsur M, Levitz R, Kaufmann G. 1987. Bacteriophage T4 anticodon nuclease, polynucleotide kinase and RNA ligase reprocess the host lysine tRNA. EMBO J 6: 2499-2503.

Bowman CM, Dahlberg JE, Ikemura T, Konisky J, Nomura M. 1971. Specific inactivation of $16 \mathrm{~S}$ ribosomal RNA induced by colicin E3 in vivo. Proc Natl Acad Sci 68: 964-968. 
Chan C, Zhou C, Brunzelle JS, Huang RH. 2009a. Structural and biochemical insights into $2^{\prime}$-O-methylation at the $3^{\prime}$-terminal nucleotide of RNA by Hen1. Proc Natl Acad Sci 106: 17699-17704.

Chan CM, Zhou C, Huang RH. 2009b. Reconstituting bacterial RNA repair and modification in vitro. Science 326: 247.

El Omari K, Ren J, Bird LE, Bona MK, Klarmann G, LeGrice SF, Stammers DK. 2006. Molecular architecture and ligand recognition determinants for T4 RNA ligase. J Biol Chem 281: 1573-1579.

Endo Y, Wool IG. 1982. The site of action of $\alpha$-sarcin on eukaryotic ribosomes. The sequence at the $\alpha$-sarcin cleavage site in $28 \mathrm{~S}$ ribosomal ribonucleic acid. J Biol Chem 257: 9054-9060.

Fando JL, Alaba I, Escarmis C, Fernandez-Luna JL, Mendez E, Salinas M. 1985. The mode of action of restrictocin and mitogillin on eukaryotic ribosomes. Inhibition of brain protein synthesis, cleavage and sequence of the ribosomal RNA fragment. Eur $J$ Biochem 149: 29-34.

Galburt EA, Pelletier J, Wilson G, Stoddard BL. 2002. Structure of a tRNA repair enzyme and molecular biology workhorse: T4 polynucleotide kinase. Structure 10: 1249-1260.

Ho CK, Shuman S. 2002. Bacteriophage T4 RNA ligase 2 (gp24.1) exemplifies a family of RNA ligases found in all phylogenetic domains. Proc Natl Acad Sci 99: 12709-12714.

Jain R, Shuman S. 2010. Bacterial Hen1 is a $3^{\prime}$ terminal RNA ribose 2'-O-methyltransferase component of a bacterial RNA repair cassette. RNA 16: 316-323.

Jain R, Shuman S. 2011. Active site mapping and substrate specificity of bacterial Hen1, a manganese-dependent 3 ' terminal RNA ribose 2'O-methyltransferase. RNA 17: 429-438.

Keppetipola N, Nandakumar J, Shuman S. 2007. Reprogramming the tRNA-splicing activity of a bacterial RNA repair enzyme. Nucleic Acids Res 35: 3624-3630.

Lee SR, Collins K. 2005. Starvation-induced cleavage of the tRNA anticodon loop in Tetrahymena thermophila. J Biol Chem 280: 42744-42749.

Lu J, Huang B, Esberg A, Johansson MJ, Bystrom AS. 2005. The Kluyveromyces lactis $\gamma$-toxin targets tRNA anticodons. RNA 11: $1648-1654$

Martins A, Shuman S. 2004a. An RNA ligase from Deinococcus radiodurans. J Biol Chem 279: 50654-50661.
Martins A, Shuman S. 2004b. Characterization of a baculovirus enzyme with RNA ligase, polynucleotide 5'-kinase, and polynucleotide $3^{\prime}$ phosphatase activities. J Biol Chem 279: 18220-18231.

Martins A, Shuman S. 2005. An end-healing enzyme from Clostridium thermocellum with $5^{\prime}$ kinase, 2',3' phosphatase, and adenylyltransferase activities. RNA 11: 1271-1280.

Nandakumar J, Schwer B, Schaffrath R, Shuman S. 2008. RNA repair: an antidote to cytotoxic eukaryal RNA damage. Mol Cell 31: 278286.

Neubauer C, Gao YG, Andersen KR, Dunham CM, Kelley AC, Hentschel J, Gerdes K, Ramakrishnan V, Brodersen DE. 2009. The structural basis for mRNA recognition and cleavage by the ribosome-dependent endonuclease RelE. Cell 139: 1084-1095.

Ogawa T, Tomita K, Ueda T, Watanabe K, Uozumi T, Masaki H. 1999. A cytotoxic ribonuclease targeting specific transfer RNA anticodons. Science 283: 2097-2100.

Pedersen K, Zavialov AV, Pavlov MY, Elf J, Gerdes K, Ehrenberg M. 2003. The bacterial toxin RelE displays codon-specific cleavage of mRNAs in the ribosomal A site. Cell 112: 131-140.

Simpson L, Sbicego S, Aphasizhev R. 2003. Uridine insertion/deletion RNA editing in trypanosome mitochondria: a complex business. RNA 9: 265-276.

Thompson DM, Lu C, Green PJ, Parker R. 2008. tRNA cleavage is a conserved response to oxidative stress in eukaryotes. RNA 14: 2095-2103.

Tomita K, Ogawa T, Uozumi T, Watanabe K, Masaki H. 2000. A cytotoxic ribonuclease which specifically cleaves four isoaccepting arginine tRNAs at their anticodon loops. Proc Natl Acad Sci 97: $8278-8283$.

Winther KS, Gerdes K. 2011. Enteric virulence associated protein VapC inhibits translation by cleavage of initiator tRNA. Proc Natl Acad Sci 108: 7403-7407.

Xu H, He WJ, Liu WY. 2004. A novel ribotoxin with ribonuclease activity that specifically cleaves a single phosphodiester bond in rat $28 \mathrm{~S}$ ribosomal RNA and inactivates ribosome. Arch Biochem Biophys 427: 30-40.

Yamasaki S, Ivanov P, Hu GF, Anderson P. 2009. Angiogenin cleaves tRNA and promotes stress-induced translational repression. J Cell Biol 185: 35-42. 

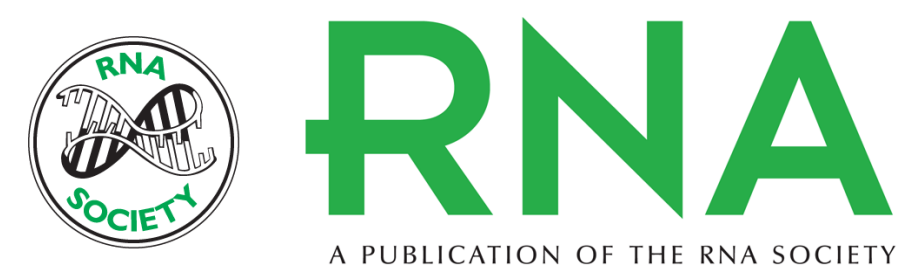

A PUBLICATION OF THE RNA SOCIETY

\title{
Probing the substrate specificity of the bacterial Pnkp/Hen1 RNA repair system using synthetic RNAs
}

\author{
Can Zhang, Chio Mui Chan, Pei Wang, et al.
}

RNA 2012 18: 335-344 originally published online December 21, 2011

Access the most recent version at doi:10.1261/rna.030502.111

\section{Supplemental http://rnajournal.cshlp.org/content/suppl/2011/12/12/rna.030502.111.DC1 Material}

References This article cites 27 articles, 19 of which can be accessed free at: http://rnajournal.cshlp.org/content/18/2/335.full.html\#ref-list-1

\section{License}

Email Alerting Receive free email alerts when new articles cite this article - sign up in the box at the Service top right corner of the article or click here. 